\title{
ACRL meetings in Washington
}

\section{A tentative schedule for ALA's Midwinter Meeting,}

January 6-12.

\section{ACRL BOARD OF DIRECTORS}

First meeting: Sunday, January 8, 2:00-5:30 p.m. Second meeting: Tuesday, January 10, 2:00-5:30 p.m.

Luncheon and orientation: Friday, January 6, 12:30-5:30 p.m.

Joint orientation and strategic planning: Saturday, January 7, 9:30 a.m.-12:30 p.m.

\section{ACRL DIVISIONAL COMMITTEES}

Academic Library Statistics: Saturday, January 7, 8:00-9:00 a.m.; Monday, January 9, 8:00-9:00 a.m.

Academic or Research Librarian of the Year Award: Monday, January 9, 2:00-5:30 p.m. ${ }^{*}$

Academic Status: Sunday, January 8, 9:00-11:00 a.m.; Monday, January 9, 9:00-11:00 a.m.; Tuesday, January 10, 9:00-11:00 a.m.

ACRL/AECT Joint Committee: Friday, January 6, 9:00 a.m. $-5: 00$ p.m.

ACRL and Social Responsibility Task Force: Saturday, January 7, 9:00-11:00 a.m.

Appointments and Nominations: Saturday, January 7, 2:00-4:00 p.m.; Monday, January 9, 9:30-11:00 a.m. *

Atkinson Award: Sunday, January 8, 9:30-11:00 a.m. ${ }^{*}$

Audiovisual: Sunday, January 8, 9:00-11:00 a.m.; Monday, January 9, 9:00-11:00 a.m.

Budget and Finance: Saturday, January 7, 9:30 a.m.-12:30 p.m., 2:00-5:30 p.m.; Sunday, January 8, 8:00 a.m.-12:30 p.m.; Monday, January 9 , 2:00-5:30 p.m.; Tuesday, January $10,9: 30$ a.m. $-12: 30$ p.m.

${ }^{*}$ Denotes a closed meeting.
Budget and Finance-Financial Plan Hearing: Sunday, January 8, 8:00-10:00 p.m.

Conference Program Planning-Chicago, 1990: Tuesday, January 10, 11:30 a.m.-12:30 p.m.

Constitution and Bylaws: Saturday, January 7, 8:00-11:00 a.m.; Monday, January 9, 8:00-11:00 a.m.

Continuing Education Courses Advisory: Sunday, January 8, 8:00 a.m.-12:30 p.m.

Copyright: Monday, January 9, 9:00-11:00 a.m.

Doctoral Dissertation Fellowship: Saturday, January 7, 2:00-4:00 p.m.*

Financial Development Task Force: Monday, January 9, 9:30 a.m.-12:30 p.m.

Guidelines for Extended Campus Library Services Task Force: Saturday, January 7, 2:00-4:00 p.m.; Sunday, January 8, 8:00-10:00 p.m.; Tuesday, January 10, 9:00-11:00 a.m.

International Relations Task Force: Saturday, January 7, 8:00-11:00 a.m.

K.G. Saur Award: Monday, January 9, 8:00-11:00 a.m.*

Legislation: Sunday, January 8, 11:30 a.m.-12:30 p.m.; Monday, January 9, 9:30-11:00 a.m.

Librarians as Instructors Task Force: Monday, January 9, 8:00-11:00 a.m., 8:00-10:00 p.m.

Library Access Task Force: Saturday, January 7, 4:30-5:30 p.m.; Monday, January 9, 9:00-11:00 a.m.

Library School Curriculum Task Force: Monday, January 9, 8:30 a.m.-12:30 p.m.

Membership: Monday, January 9, 9:30 a.m. $-12: 30$ p.m.

Minority Recruitment Task Force: Monday, January 9, 9:30-11:00 a.m.

National Conference Executive: Saturday, January 7, 12:30-2:00 p.m.; Monday, January 9, 8:00 a.m. $-12: 30$ p.m. 
Performance Measures: Saturday, January 7, 2:00-4:00 p.m.; Monday, January 9, 9:00-11:00 a.m.

Planning: Saturday, January 7, 2:00-5:30 p.m.; Monday, January 9, 9:30 a.m.-12:30 p.m.

Professional Association Liaison: Saturday, January 7, 2:00-4:00 p.m.; Monday, January 9, 9:00-11:00 a.m.

Professional Education: Sunday, January 8, 9:30 a.m. $-12: 30$ p.m.

Professional Ethics Task Force: Monday, January 9, 2:00-4:00 p.m.

Publications: Monday, January 9, 2:00-4:00 p.m.; Tuesday, January 10, 9:30 a.m.-12:30 p.m.

Research: Sunday, January 8, 9:30 a.m.-12:30 p.m.; Monday, January 9, 9:30 a.m.-12:30 p.m.

Samuel Lazerow Fellowship: Monday, January 9, 8:00-9:00 a.m.*

Sources of Revenue in Academic Libraries Task Force: Saturday, January 7, 2:00-4:00 p.m.; Tuesday, January 10, 9:30-11:00 a.m.

Standards and Accreditation: Saturday, January 7, 9:00-11:00 a.m.; Monday, January 9, 9:00-11:00 a.m.

\section{ACRL CHAPTERS COUNCIL}

Business: Sunday, January 8, 8:30 a.m.-12:30 p.m.

Strategic Planning: Friday, January 6, 8:00-10:00 p.m.

Presidential Candidate Forum and Reception: Saturday, January 7, 8:00-10:00 p.m.

\section{ACRL DISCUSSION GROUPS}

Australian Studies: Sunday, January 8, 4:30-5:30 p.m.

Black Studies: Saturday, January 7, 2:00-4:00 p.m.

Canadian Studies: Saturday, January 7, 2:00-5:30 p.m.

Electronic Library Development in Academic Libraries: Saturday, January 7, 11:30 a.m. $-12: 30$ p.m.

English and American Literature: Monday, January 9, 9:00-11:00 a.m.

English and American Literature-Program Planning: Monday, January 9, 8:00-9:00 a.m.

Extended Campus Library Service: Monday, January 9, 9:30-11:00 a.m.

Fee-Based Information Service Centers in Academic Libraries: Sunday, January 8, 9:00-11:00 a.m.; Monday, January 9, 9:00-11:00 a.m.

Heads of Reader/Public Services: Sunday, January 8, 4:30-6:00 p.m.

Journal Costs in Academic and Research Libraries: Sunday, January 8, 9:30-11:00 a.m.

Librarians of Library Science Collections-Tour of SLA Library: Monday, January 9, 9:30-11:00 a.m., Special Libraries Association, 1700 18th St., N.W.

Microcomputer Services in Academic Libraries: Sunday, January 8, 2:00-4:00 p.m.; Tuesday, January 10, 9:00-11:00 a.m.

Personnal Administrators and Staff Development Officers: Friday, January 6, 1:00-5:00 p.m.; Saturday, January 7, 9:30-11:00 a.m.; Sunday, January 8, 9:30-11:30 a.m.; Monday, January 9, 9:30-11:00 a.m.

Public Relations in Academic Libraries: Sunday, January 8, 2:00-5:30 p.m.

Research: Monday, January 9, 9:30-11:00 a.m.

Undergraduate Librarians: Monday, January 9 , 2:00-4:00 p.m.

\section{ACRL EDITORIAL BOARDS}

College \& Research Libraries: Sunday, January 8, 9:00-11:00 a.m.

College \& Research Libraries News: Sunday, January 8, 8:00-9:00 a.m.

Publications in Librarianship: Monday, January 9, 8:30-11:00 a.m.

Rare Books \& Manuscripts Librarianship: Saturday, January 7, 2:00-4:00 p.m.

\section{ACRL SECTIONS}

\section{Activity Sections Council}

Meeting: Tuesday, January 10, 4:30-5:30 p.m. Section Officers/Executive Committee Luncheon: Monday, January 9, 12:30-2:00 p.m.

\section{Anthropology and Sociology Section}

Executive: Saturday, January 7, 2:00-4:00 p.m.; Monday, January 9, 2:00-4:00 p.m.

Bibliography: Saturday, January 7, 9:00-11:00 a.m.; Sunday, January 8, 2:00-4:00 p.m.

Conference Program Planning-Chicago, 1990: Saturday, January 7, 8:00-9:00 a.m.; Monday, January 9, 8:00-9:00 a.m.

Conference Program Planning-Dallas, 1989: Saturday, January 7, 8:00-9:00 a.m.; Monday, January 9, 8:00-9:00 a.m.

\section{WESS discussion group for smaller collections}

A College and Medium-Sized Libraries Discussion Group has been formed within the Western European Specialists Section (WESS) to promote an exchange of information and ideas on the acquisition, organization, and use of Western European materials as they relate to the special circumstances of smaller library collections. Chaired this year by David Cohen, College of Charleston, Charleston, SC 29424, it will have its first regular meeting this Midwinter on Sunday, January 8, from 11:30 a.m. to 12:30 p.m. 
Nominating: Sunday, January 8, 9:00-11:00 a.m.; Monday, January 9, 11:30 a.m.-12:30 p.m.

Publications: Saturday, January 7, 11:30 a.m.-12:30 p.m.; Monday, January 9, 9:00-11:00 a.m.

Review and Planning: Sunday, January 8 , 8:00-9:00 a.m.

\section{Art Section}

Executive: Sunday, January 8, 9:30-11:00 a.m.

Committees: Saturday, January 7, 2:00-4:00 p.m.

Membership: Sunday, January 8, 11:30 a.m. $-12: 30$ p.m.

Technology in the Arts: Monday, January 9, 9:30-11:00 a.m.

\section{Asian and African Section}

Executive: Sunday, January 8, 9:00-11:00 a.m.

\section{Bibliographic Instruction Section}

Executive: Saturday, January 7, 8:00-9:00 a.m.; Tuesday, January 10, 11:30 a.m.-12:30 p.m.; Wednesday, January 11, 9:00-11:00 a.m.

BIS dinner: Friday, January 6, 6:30-9:00 p.m., China Inn, 631 H St., N.W.

Advisory Council: Saturday, January 7, 9:30-11:30 a.m.; Monday, January 9, 9:30 a.m. $-12: 30$ p.m.

Communication: Saturday, January 7, 2:00-4:00 p.m.; Sunday, January 8, 2:00-4:00 p.m.

Computer Concerns: Sunday, January 8, 9:30 a.m.-12:30 p.m.; Tuesday, January 10, 9:00-11:00 a.m.

Conference Program Planning-Chicago, 1990: Saturday, January 7, 2:00-4:00 p.m.; Monday, January 9, 2:00-4:00 p.m.

Conference Program Planning-Dallas, 1989: Saturday, January 7, 2:00-4:00 p.m.; Tuesday, January 10, 9:00-11:00 a.m.

Conference Program Planning-Dallas, 1989Local Arrangements: Sunday, January 8, 2:00-4:00 p.m.

Continuing Education: Saturday, January 7, 2:00-4:00 p.m.; Monday, January 9, 2:00-4:00 p.m.

Cooperation Task Force: Sunday, January 8, 2:00-4:00 p.m.

Education for Bibliographic Instruction: Saturday, January 7, 2:00-4:00 p.m.; Sunday, January $8,9: 30$ a.m. $-12: 30$ p.m.

Miriam Dudley BI Librarian of the Year Award: Saturday, January 7, 11:30 a.m.-12:30 p.m.; Monday, January 9, 2:00-4:00 p.m.*

Nominating: Sunday, January 8, 9:30 a.m. $-12: 30$ p.m.*

Planning: Monday, January 9, 2:00-4:00 p.m.

Policy: Sunday, January 8, 9:30 a.m.-12:30 p.m.; Tuesday, January 10, 9:30-11:00 a.m.

Research: Saturday, January 7, 4:30-5:30 p.m.

Think Tank Steering: Saturday, January 17, 2:00-4:00 p.m.; Monday, January 9, 2:00-4:00 p.m.

\section{College Libraries Section}

Executive: Saturday, January 7, 9:00 a.m.-12:30 p.m.; Tuesday, January 10, 11:30 a.m.-12:30 p.m.

CLIP Notes: Monday, January 9, 2:00-4:00 p.m.

Conference Program Planning-Chicago, 1990: Saturday, January 7, 4:30-5:30 p.m.

Conference Program Planning-Dallas, 1989: Saturday, January 7, 4:30-5:30 p.m.

Discussion Group: Sunday, January 8, 2:00-4:00 p.m.

Historical Commission: Saturday, January 7, 2:00-5:30 p.m.; Monday, January 9, 8:00 a.m. $-12: 30$ p.m.

National Advisory Council Steering: Saturday, January 7, 2:00-4:00 p.m.

Newsletter: Saturday, January 7, 2:00-5:30 p.m.

Real Income: Monday, January 9, 2:00-4:00 p.m.

Selection Guidelines: Sunday, January 8, 9:30-11:00 a.m.

Standards: Saturday, January 7, 2:00-4:00 p.m.; Tuesday, January 10, 9:00-11:00 a.m.

\section{Community and Junior College Libraries Section}

All committees: Sunday, January 8, 8:30 a.m. $-12: 30$ p.m.

\section{Education and Behavioral Sciences Section}

Executive: Tuesday, January 10, 8:00-11:00 a.m. Bibliographic Instruction for Educators: Sunday, January 8, 9:00 a.m.-12:30 p.m.

Conference Program Planning-Chicago, 1990: Saturday, January 7, 8:00-10:00 a.m., 2:00-4:00 p.m.

Problems of Access and Control of Education Materials: Sunday, January 8, 2:00-5:30 p.m.

Psychology/Psychiatry: Monday, January 9, 8:00-11:00 a.m., 2:00-4:00 p.m.

Publications: Sunday, January 8, 8:00-9:00 a.m.

\section{Law and Political Science Section}

Executive: Saturday, January 7, 9:00-11:00 a.m.

Business: Monday, January 9, 2:00-4:00 p.m.

Bibliographies: Saturday, January 7, 2:00-4:00 p.m.

Conference Program Planning-Chicago, 1990: Sunday, January 8, 9:00-11:00 a.m.

Conference Program Planning-Dallas, 1989: Monday, January 9, 9:00-11:00 a.m.

Conference Program Planning-Dallas, 1989: Sunday, January 8, 2:00-4:00 p.m.; Monday, January 9, 9:00-11:00 a.m.

Review and Planning: Monday, January 9, 9:00-11:00 a.m.

\section{Rare Books and Manuscripts Section}

Executive: Sunday, January 8, 2:00-5:30 p.m.; Monday, January 9, 9:30 a.m.-12:30 p.m.

Cambridge Conference Planning: Sunday, January 8, 2:00-4:00 p.m.

Conference Program Planning-Chicago, 1990: 


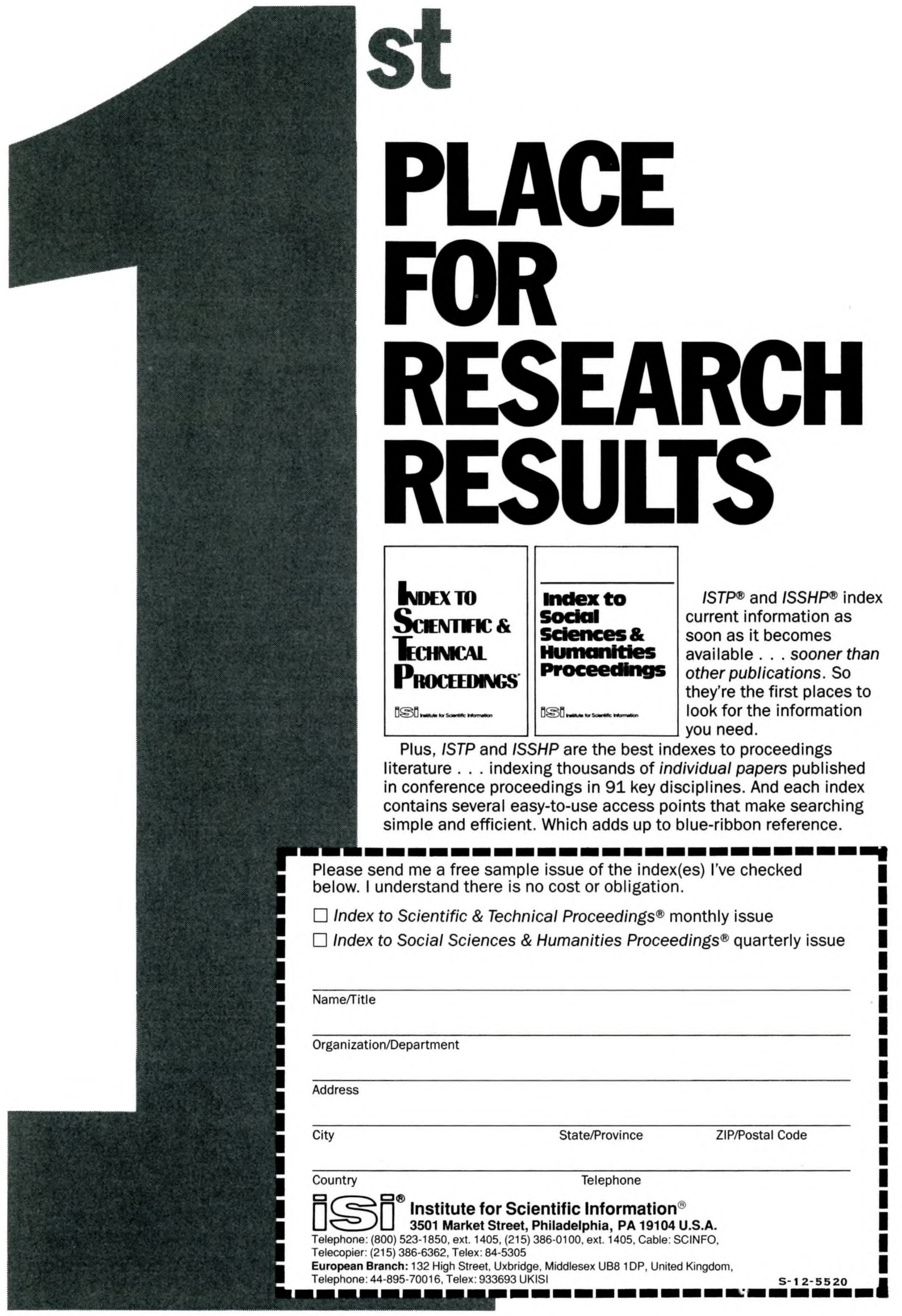


Sunday, January 8, 9:00-11:00 a.m.

Conference Program Planning-Dallas, 1989: Sunday, January 8, 11:30 a.m.-12:30 p.m.

Conservator's Collations: Sunday, January 8, 9:00-11:00 a.m.

Curators and Conservators Discussion Group: Saturday, January 7, 11:30 a.m.-12:30 p.m.

Developing Guidelines for Borrowing Special Collection Materials for Exhibition: Saturday, January 7, 9:00-11:00 a.m.

Education and Professional Development: Saturday, January 7, 2:00-4:00 p.m.

Ethical Standards Review: Sunday, January 8, 9:00-11:00 a.m.

Exhibition Catalogues Awards: Saturday, January 7, 9:00-11:00 a.m.; Sunday, January 8, 2:00-4:00 p.m.*

Gifts and Appraisals Brochure: Sunday, January 8, 9:00-11:00 a.m.

GODORT/MAGERT Joint Committee: Sunday, January 8, 11:30 a.m.-12:30 p.m.

Guidelines on Archives and Manuscripts Review: Saturday, January 7, 9:00-11:00 a.m.

Information Exchange: Saturday, January 7, 4:30-5:30 p.m.

Legislative Information: Saturday, January 7, 9:00-11:00 a.m.

Literary Rights: Sunday, January 8, 11:30 a.m. $-12: 30$ p.m.

Manuscripts and Other Formats: Saturday, January 7, 8:00-10:00 p.m.

MARC for Special Collections: Sunday, January 8, 2:00-4:00 p.m.

Nominating: Saturday, January 7, 8:00-10:00 p.m.*

Orientation Manual: Saturday, January 7, 11:30 a.m. $-12: 30$ p.m.

Preconference Program Planning-Dallas, 1989: Saturday, January 7, 2:00-4:00 p.m.

Publications: Sunday, January 8, 9:00-11:00 a.m.

Records Retention: Saturday, January 7, 9:00-11:00 a.m.

Security: Saturday, January 7, 9:00-11:00 a.m.

Standards: Saturday, January 7, 2:00-4:00 p.m.; Sunday, January 8, 2:00-4:00 p.m., 8:00-10:00 p.m.

Statistical Survey Feasibility: Saturday, January 7 , 2:00-4:00 p.m.

\section{Science and Technology Section}

Council: Saturday, January 7, 8:30-11:00 a.m.; Tuesday, January 10, 9:00-11:00 a.m.

Comparison of Science and Engineering Libraries: Saturday, January 7, 9:30-11:00 a.m.

Conference Proceedings-Bibliographic Access: Saturday, January 7, 9:30-11:00 a.m.

Conference Proceedings-Importance of Proceedings: Saturday, January 7, 9:30-11:00 a.m.

Conference Program Planning-Chicago, 1990: Monday, January 9, 8:30 a.m.-12:30 p.m.

Conference Program Planning-Dallas, 1989: Sunday, January 8, 8:30 a.m.-12:30 p.m.
Discussion Group: Saturday, January 7, 2:00-4:00 p.m.

Legislation: Saturday, January 7, 9:30-11:00 a.m.

Membership: Sunday, January 8, 9:00-11:00 a.m.

Newsletter: Sunday, January 8, 2:00-4:00 p.m.

Oberly Award: Sunday, January 8, 9:00-11:00 a.m.*

Planning: Sunday, January 8, 2:00-4:00 p.m.; Monday, January 9, 9:00-11:00 a.m.

Publisher Relations: Monday, January 9, 9:00-11:00 a.m.

Science Databases Discussion Group: Monday, January 9, 2:00-4:00 p.m.

Subject and Bibliographic Access to Scientific and Technical Information: Sunday, January 8, 2:00-4:00 p.m.

\section{Slavic and East European Studies Section}

Executive: Monday, January 9, 9:30-11:00 a.m.

Automated Bibliographic Control: Sunday, January 8, 9:30-11:00 a.m.

Conference Program Planning-Chicago, 1990: Monday, January 9, 8:00-9:00 a.m.

Conference Program Planning-Dallas, 1989: Sunday, January 8, 11:30 a.m.-12:30 a.m.

Continuing Education on Slavic and East European Librarianship in North America: Sunday, January 8, 2:00-4:00 p.m.

Newsletter: Sunday, January 8, 4:30-5:30 p.m.

\section{University Libraries Section}

Steering: Saturday, January 7, 9:30-11:00 a.m.; Monday, January 9, 9:00-11:00 a.m.

Conference Program Planning-Dallas, 1989: Friday, January 6, 8:00-10:00 p.m.; Sunday, January $8,9: 30-11: 00$ a.m.

Organization and Bylaws: Sunday, January 8 , 9:30-11:00 a.m.; Monday, January 9, 2:00-4:00 p.m.

\section{Western European Specialists Section}

Executive: Saturday, January 7, 4:30-5:30 p.m.; Tuesday, January 10, 2:00-4:00 p.m.

Classical, Medieval and Renaissance Discussion Group: Saturday, January 7, 2:00-4:00 p.m.

College and Medium-Sized Libraries Discussion Group: Sunday, January 8, 11:30 a.m.-12:30 p.m.

Conference Program Planning-Chicago, 1990: Monday, January 9, 8:00-9:00 a.m.

\section{Gelman Library reunion}

Are you one of Gelman's alumni? Are you interested in getting reacquainted with former colleagues? Want to see what we've accomplished since the last time you visited? Please accept our invitation to join us on Monday, January 9 , at a reception from $5: 30-7: 30$ p.m. in Gelman Room 202. We look forward to seeing you there. 
Conference Program Planning-Dallas, 1989: Tuesday, January 10, 8:00-9:00 a.m.

Continuing Education: Sunday, January 8, 2:00-4:00 p.m.

Florence Conference Proceedings: Saturday, January 7, 2:00-4:00 p.m.

General Discussion Group: Monday, January 9, 2:00-4:00 p.m.

Germanists Discussion Group: Sunday, January 8, 9:30-11:00 a.m.

Martinus Nijhoff Award Jury: Sunday, January 8, 8:00-10:00 p.m. *

Nominating: Sunday, January 8, 8:00-9:00 a.m. ${ }^{*}$

Publications: Tuesday, January 10, 9:30-11:00 a.m.

Publications-Directory: Tuesday, January 10, 8:00-9:00 a.m.

Publications-Newsletter: Monday, January 9, 11:30 a.m.-12:30 p.m.

Research and Planning: Monday, January 9, 9:30-11:00 a.m.

\section{Women's Studies Section}

Executive: Friday, January 6, 2:00-4:00 p.m.

Business: Monday, January 9, 9:30-11:00 a.m.

Communications: Saturday, January 7, 2:00-4:00 p.m.

Conference Program Planning-Dallas, 1989: Saturday, January 7, 9:00-11:00 a.m.

National Conference Planning-Cincinnati: Saturday, January 7, 2:00-4:00 p.m.

Newsletter: Saturday, January 7, 4:30-5:30 p.m.
Technical Services: Saturday, January 7, 9:00-11:00 a.m.

\section{Popular culture and libraries}

A new ACRL discussion group will examine the relationships (or lack thereof) between libraries and popular culture materials. Popular culture materials include genre fiction, comic books and strips, pulp magazines, dime novels, show business memorabilia, trading cards, greeting cards, big little books, and similar everyday ephemera that can be considered integral to American life, yet which the library profession has traditionally considered trash unworthy of collection. The last two decades have seen a steady increase in the scholarly and aesthetic evaluation of such materials.

The discussion group will consider the problems involved in meeting the needs of a popular culture clientele, including identifying existing collections, establishing cataloging standards, preservation, collection development, reviewing new and often overlooked materials, and educating the general library profession to recognize their value.

Interested parties wanting additional information should contact Allen Ellis, W. Frank Steely Library, Northern Kentucky University, Highland Heights, KY 41076.

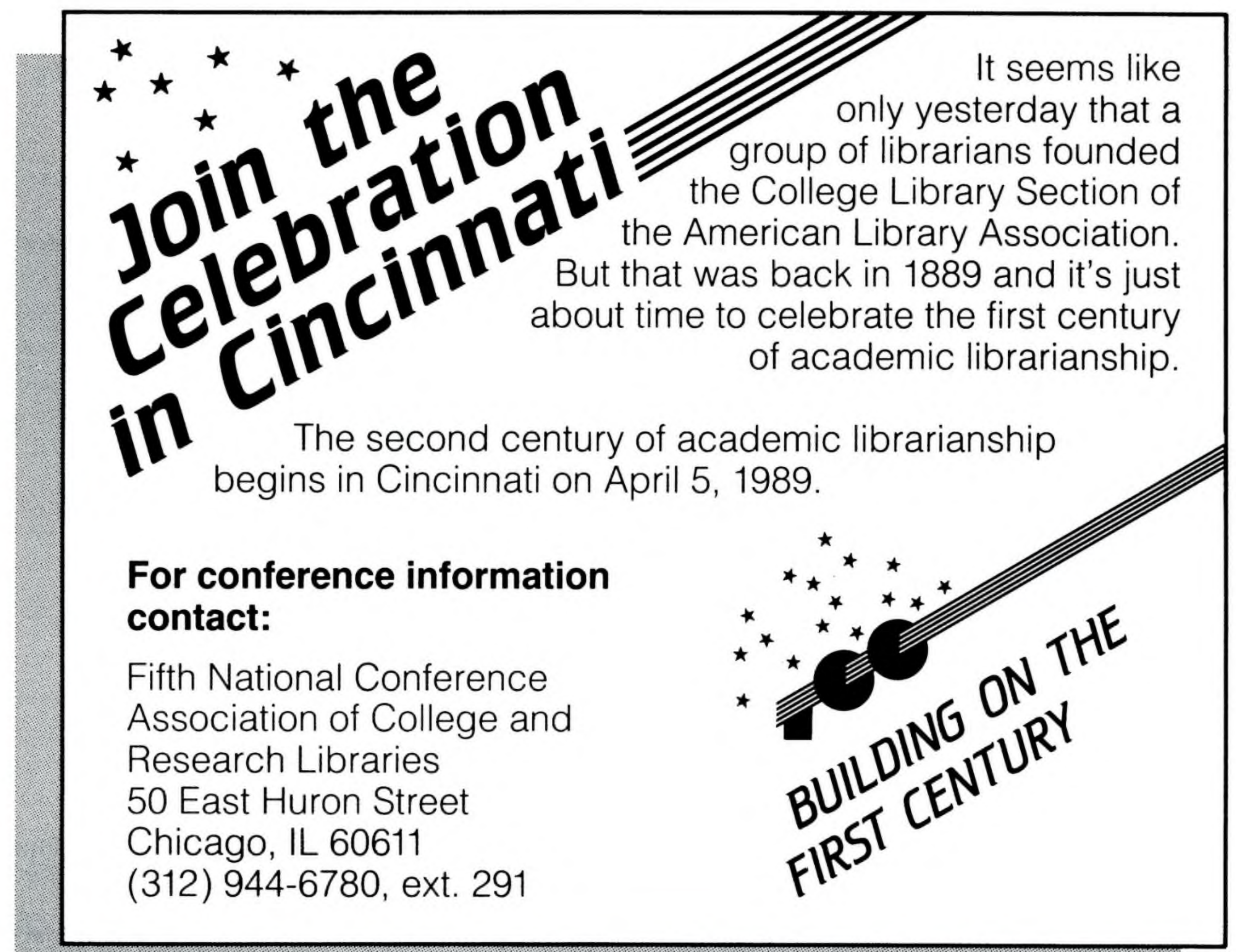




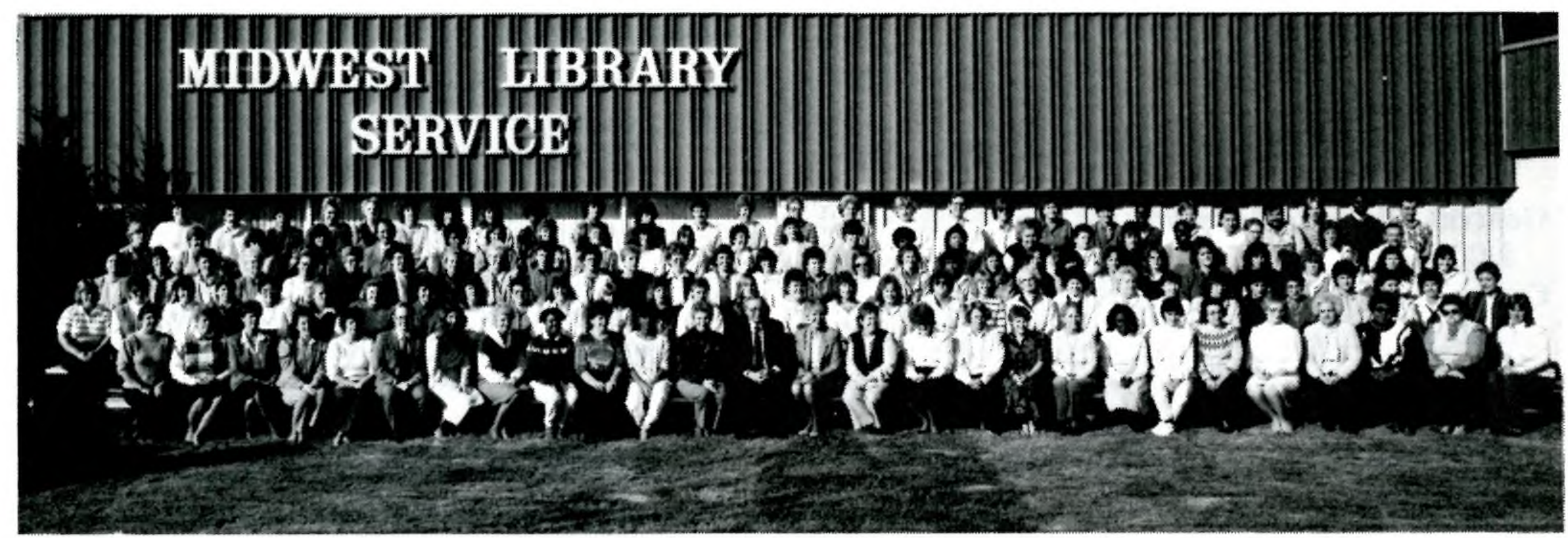

\section{MLS-160 People \\ Dedicated to Providing Service to Libraries}

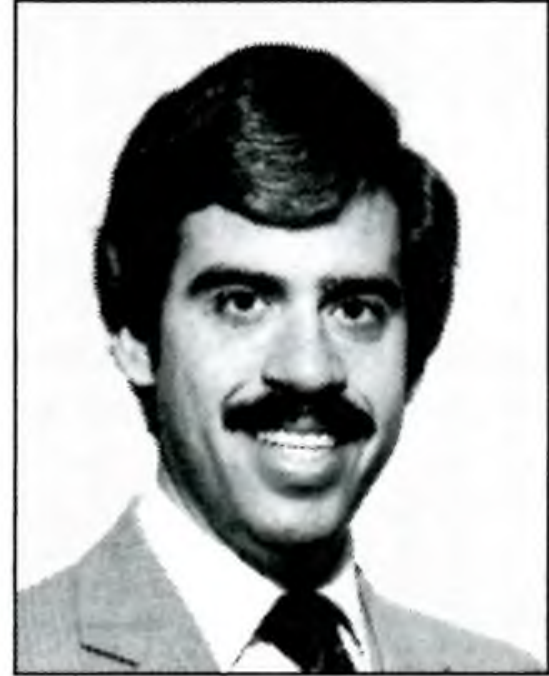

Jay Askuvich General Sales Manager

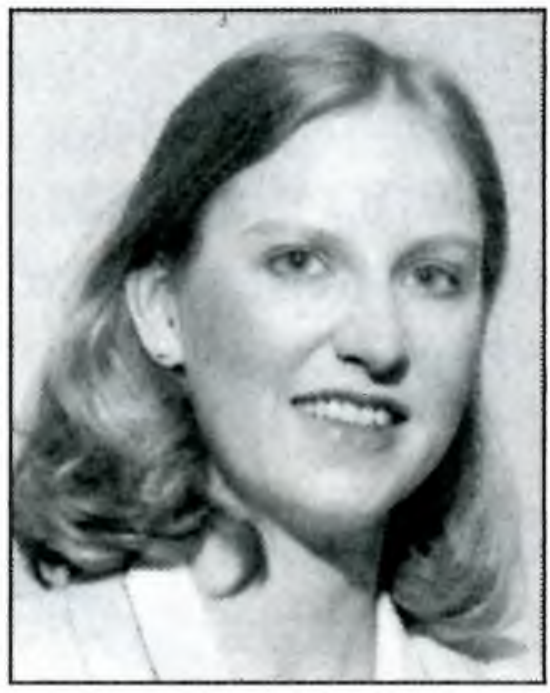

Eva Mahanna Southwest

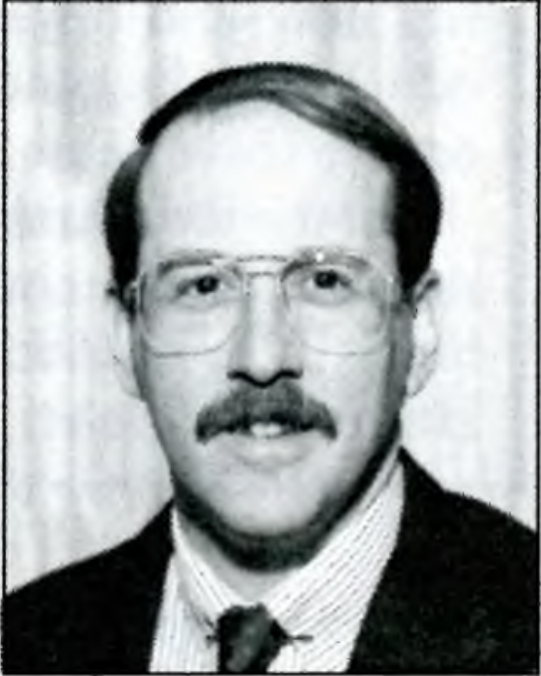

Scott Schmidt

Midwest

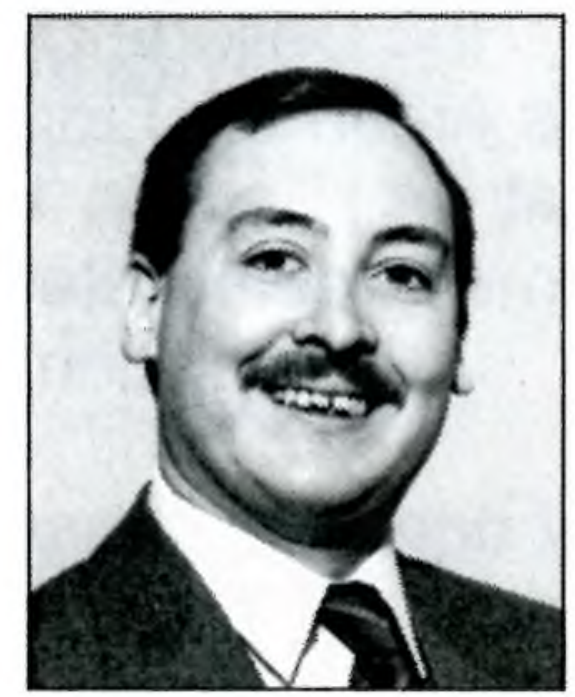

Forrest Link Northeast

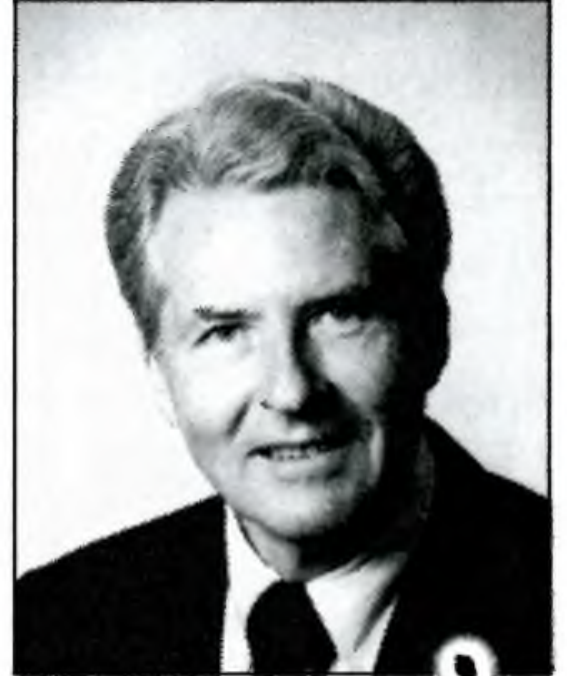

Carl Dorr

Southeast

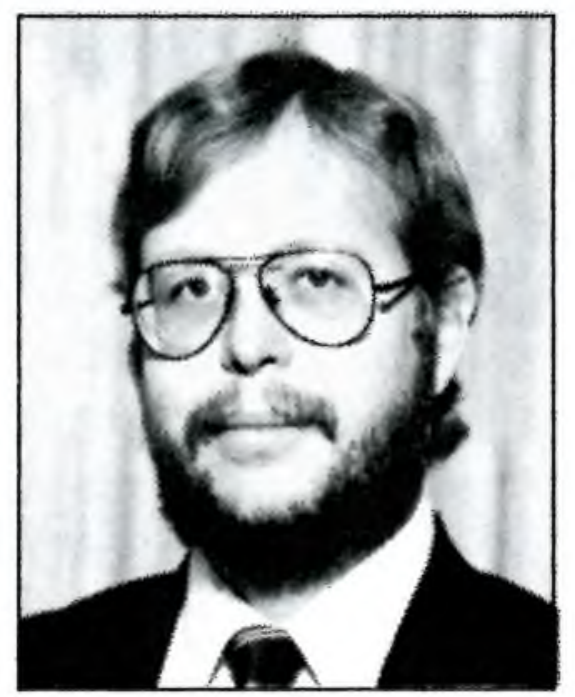

Kim Anderson

Mountain Plains

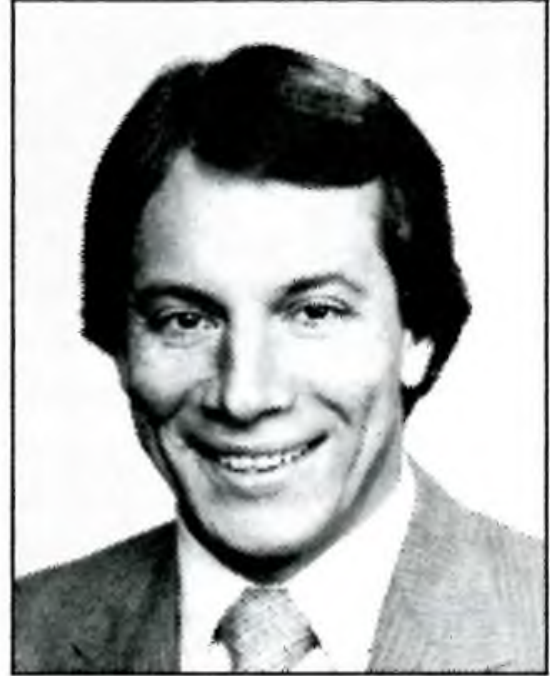

Lawrence Nagel West

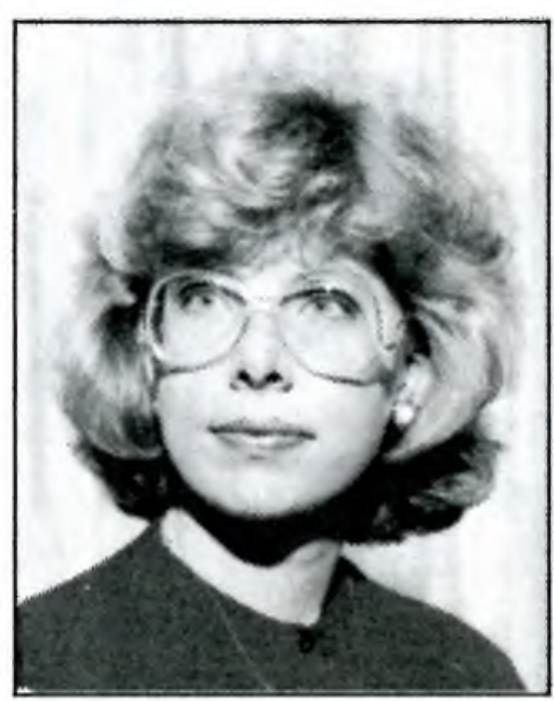

Lorraine Best

Canada

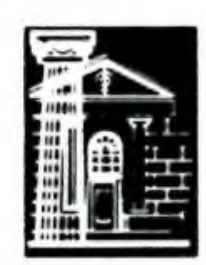

\section{Midwest Library Service}

11443 St. Charles Rock Road

Bridgeton, MO 63044, USA

Call toll-free 1-800-325-8833

Missouri librarians call toll-free 1-800-392-5024

Canadian librarians call toll-free 1-800-527-1659 